believe this for some developmental domains $(p<0.05)$. Overall, $32 \%$ of parents had sought information on child development and behaviour from childcare providers. Independent predictors of using childcare providers as a resource included having children under the age of 6 years ( $O R=1.65,95 \%$ CI 1.11 to 2.46$)$, having children in care for more than $6 \mathrm{~h}$ per week $(\mathrm{OR}=19.88,95 \% \mathrm{CI} 11.51$ to 34.35$)$, and being unmarried (OR=2.08, 95\% CI 1.16 to 3.73$)$.

Conclusions Among Alberta adults who recently interacted with children, there was support for public funding of childcare. Adults recognised that childcare providers play a critical role in supporting optimal child development and their beliefs about the need for education among childcare providers are in line with research evidence in the area. Policy and decision makers may find this information helpful in allocating resources to promote child development.

\section{P1-360 INCIDENCE OF STROKE FOLLOWING HERPES ZOSTER AMONG POPULATION 50 YEARS AND OLDER}

doi:10.1136/jech.2011.142976f.52

H F Tseng, ${ }^{*}$ N Smith, S Jacobsen. Kaiser Permanete, Pasadena, California, USA

Introduction Although varicella zoster virus (VZV)-induced vasculopathy and stroke after herpes zoster $(\mathrm{HZ})$ attacks have been previously reported, data regarding stroke frequency after zoster attacks are lacking. This study investigates the risk of stroke after $\mathrm{HZ}$ in a general population age 50 years and older.

Methods This is a retrospective cohort study of $\mathrm{HZ}$ cases who had received treatment for HZ from 1 January 2007 to 31 October 2010 at Kaiser Permanente Southern California. These cases had no records of stroke within 1 year prior to their index $\mathrm{HZ}$ diagnosis date. The comparison cohort included subjects who had no HZ during the same period and were matched 1:1 to the HZ cohort on age, date of $\mathrm{HZ}$ diagnosis, and the setting of medical care. Incident cases of stroke were identified from hospitalisation records with primary diagnosis as stroke.

Results There were 227 stroke cases in the HZ cohort and 224 in the comparison cohort. The 1-year Kaplan-Meier cumulative incidence of stroke was $0.88 \%$ (95\% CI $0.77 \%$ to $1.00 \%$ ) for the HZ cohort and $0.89 \%$ (95\% CI $0.78 \%$ to $1.00 \%$ ) for the comparison cohort ( $\mathrm{p}$ value: 0.98). The HR of stroke after any HZ or herpes zoster ophthalmicus, after controlling for sex, race, heart diseases, diabetes, lung diseases, kidney diseases, liver diseases, hypertension, and demential status was 1.11 (95\% CI 0.92 to 1.33 ) and 3.33 (95\% CI 1.05 to 10.53), respectively.

Conclusions These data suggest that the risk of stroke following herpes zoster was increased in only those with ophthalmic involvement and not in subjects with $\mathrm{HZ}$ involved in distant dermatomes.

\section{P1-361 HISTORY OF CHILD ABUSE AND SELF-INJURIOUS BEHAVIOUR}

doi:10.1136/jech.2011.142976f.53

${ }^{1} \mathrm{~S}$ Tsuboi, ${ }^{*}{ }^{1} \mathrm{R}$ Ae, ${ }^{1} \mathrm{~T}$ Kojo, ${ }^{2} \mathrm{H}$ Yoshida, ${ }^{1} \mathrm{Y}$ Nakamura. ${ }^{1}$ Jichi Medical University, Shimotsuke, Tochigi, Japan; ${ }^{2}$ Harvard School of Public Health, Boston, Massachusetts, USA

Introduction It is not well known what kind of health problems is associated with the history of child abuse. The aim of this study was to observe the current situation of child abuse in Japan, and to confirm our hypothesis that history of child abuse is associated with self-injurious behaviour.

Methods Data from the Lifestyle and Attitude towards Sexual Behaviour Survey were used for the secondary analysis. Using the two-stage stratified random sampling method, 3000 people (aged 16-49) were selected throughout Japan. Visit custody investigation was conducted to collect each one's data, including general characteristics (gender, age, education, occupational status, marital status, tobacco use, and alcohol consumption), history of parents' divorce, history of child abuse, and self-injurious behaviour. The $\chi 2$-test and the logistic regression analysis were used to analyse the relationship between history of child abuse and self-injurious behaviour.

Results A total of 1540 participants (51\%) were included in the analysis. Of these, 77 participants (5\%) experienced child abuse. Also 108 participants (7\%) had experience self-injurious behaviour at least once. Although $6 \%$ of the participants without the history of child abuse attempted self-injurious behaviour, $33 \%$ of the participants with the history did attempt self-injurious behaviour. History of child abuse was significantly associated with self-injurious behaviour in crude and adjusted model.

Conclusion We found that history of child abuse was associated with self-injurious behaviour. Public health workers and clinicians should pay attention to the future self-injurious behaviour in caring for child abuse.

\section{P1-362 CHARACTERISATION OF ABORTION CASES AT A PUBLIC MATERNITY IN NATAL, BRAZIL}

doi:10.1136/jech.2011.142976f.54

${ }^{1} \mathrm{~S}$ A Uchoa, ${ }^{*} \mathrm{C}$ Nilma, ${ }^{1} \mathrm{~F}$ M Celia, ${ }^{1} \mathrm{~S}$ M Helena, ${ }^{2} \mathrm{~F}$ Rudgy. ${ }^{1}$ Federal University of Rio grande do norte, Natal, Rio grande do norte, Brazil; '2State University of São Paulo, Public health school, São paulo, Brazil

Introduction Legal abortions in Brazil are restricted to risk of maternal death and rape. The aim of this study was to characterise abortion cases at a public maternity in Natal, Brazil. Method: This is a quantitative descriptive study. The population included all adolescents and young adults (1554) hospitalised as a result of illegal abortions in 2007 and 2008.

Results Abortion accounted for $98 \%$ of emergency cases; mean age was 20 years; mean gestational age was 11 weeks; 93\% denied induced abortion. The most common cause of hospitalisation was abdominal pain accompanied by haemorrhage (60\%); incomplete abortion (74.4\%); 60\% underwent curettage and 40\% manual intrauterine aspiration. There was a positive association between aspiration, higher gestational age and maternal age; one-day hospitalisation without complications or counselling (93\%).

Conclusion There is a serious public health problem with high occurrence of abortions in progress and only emergency care.

\section{P1-363 THE IMPACT OF SCHOOL CLOSURE DURING THE A/H1N1 EPIDEMIC 2009 INFLUENZA PANDEMIC AMONG SCHOOLS IN JAPAN}

doi:10.1136/jech.2011.142976f.55

${ }^{1} \mathrm{~A}$ Uda, ${ }^{*} \mathrm{M}$ Okada, ${ }^{1} \mathrm{H}$ Takahashi, ${ }^{2} \mathrm{~K}$ Motegi, ${ }^{1} \mathrm{Y}$ Wagatsuma. ${ }^{1}$ Department of Epidemiology, Graduate School of Comprehensive Human Sciences, University of Tsukuba, Tsukuba City, Japan; ${ }^{2}$ baraki Prefecture Hitachi Public Health Center, Hitachi City, Japan

Introduction In Japan, the first infected person to the influenza(A/ H1N1) was confirmed, May 2009, and spread widely, especially among children. Schools conducted various preventive actions under a general guideline by the local education board. School or class closure was one of them, which was decided by each school. However, it is not clear whether school closure is effective. The objective of the study was to examine the effectiveness of school closure. 